\title{
NITROGÊNIO, FÓSFORO E POTÁSSIO NA NUTRIÇÃO E NA PRODUÇÃO DE MUDAS DE LARANJEIRA 'VALÊNCIA', ENXERTADA SOBRE CITRUMELEIRO 'SWINGLE'1
}

\author{
RENATO DE MELLO PRADO ${ }^{2}$, DANILO EDUARDO ROZANE ${ }^{3}$, GUSTAVO SILVACAMAROTTI ${ }^{4}$, \\ MARCUS ANDRE RIBEIRO CORREIA ${ }^{5}$, WILLIAM NATALE ${ }^{6}$, JOSÉ CARLOS BARBOSA $^{7}$, AMAURI NELSON BEUTLER $^{8}$
}

RESUMO - O experimento foi conduzido em estufa telada na FCAV/Unesp Câmpus Jaboticabal-SP, durante o período de novembro de 2005 a janeiro de 2007. O estudo teve por objetivo avaliar componentes do desenvolvimento e do estado nutricional de mudas de laranjeira 'Valência', enxertada sobre citrumeleiro 'Swingle', cultivado em substrato, em função de doses de nitrogênio, fósforo e potássio. O delineamento experimental foi o inteiramente casualizado, em esquema fatorial $3^{3}+1$, sendo 3 fatores (nitrogênio, fósforo e potássio), 3 doses e uma testemunha (sem adubação), com 3 repetições. A unidade experimental foi constituída de uma muda de laranjeira por sacolas de $5 \mathrm{dm}^{-3}$, contendo $2,5 \mathrm{~kg}$ de substrato casca de Pinus e vermiculita. Os tratamentos foram constituídos pela aplicação das seguintes doses de nutrientes em $\mathrm{mg}$ por $\mathrm{dm}^{3}$ de substrato: $\mathrm{N}_{1 / 2}: 459, \mathrm{~N}_{1}: 918$ e $\mathrm{N}_{2}: 1836 ; \mathrm{P}_{1 / 2}: 92, \mathrm{P}_{1}: 184$ e $\mathrm{P}_{2}: 368 ; \mathrm{K}_{1 / 2}: 438$, $\mathrm{K}_{1}: 876$ e $\mathrm{K}_{2}: 1752$. Aos 424 dias após a semeadura, as plantas foram divididas em raízes e parte aérea para a determinação da massa da matéria seca, altura, área foliar, diâmetro do caule e conteúdo de nutrientes. As adubações com N, P e K proporcionaram maior crescimento e maior acúmulo de N, P e K na parte aérea e nas raízes das mudas de laranjeira, em substrato de casca de Pinus e vermiculita, em relação à testemunha. A dose de $459 \mathrm{mg} \mathrm{dm}^{-3} \mathrm{de} \mathrm{Ne}$ as doses de $\mathrm{Pe} \mathrm{K} 184 \mathrm{e} 876 \mathrm{mg} \mathrm{dm}^{-3}$, respectivamente, proporcionaram melhor crescimento da parte aérea das mudas; porém, na dose recomendada de $\mathrm{N}$ de $918 \mathrm{mg} \mathrm{dm}^{-3}$, ocorreu maior crescimento do sistema radicular.

Termos de indexação: N P K, estado nutricional, mudas de citros, Citrus ssp.

\section{NITROGEN, PHOSPHORUS AND POTASSIUM ON NUTRITION AND PRODUCTION OF SEEDLINGS OF 'VALENCIA' ORANGE}

ABSTRACT - The experiment was led in a protected greenhouse in the FCAV/Unesp, Jaboticabal Campus -SP, during the period of November of 2005 to January of 2007. The study objective was to evaluate the development of components and the nutritional state of seedlings of 'Valencia' orange grafted on lemon tree citrumelo 'Swingle' rootstock, in function of nitrogen, phosphorus and potassium levels. The experimental design was entirely randomized, in factorial scheme $3^{3}+1$, being 3 factors (nitrogen, phosphorus and potassium - NPK), 3 levels and a witness (without fertilization), with 3 replications. The experimental unit was constituted of a 'Valencia' sweet orange seedling grafted on lemon tree citrumelo 'Swingle' rootstock in a bag with $2,5 \mathrm{~kg}$ of substratum. The treatments were constituted of application of the following doses of nutrients in $\mathrm{mg}$ for $\mathrm{dm}^{3}$ of substratum: $\mathrm{N}_{1 / 2}: 459, \mathrm{~N}_{1}: 918$ and $\mathrm{N}_{2}: 1836 ; \mathrm{P}_{1 / 2}: 92$, $\mathrm{P}_{1}: 184$ and $\mathrm{P}_{2} 368 ; \mathrm{K}_{1 / 2}: 438, \mathrm{~K}_{1}: 876$ and $\mathrm{K}_{2}: 1752$. In the 424 days after the planting, the plants were divided in root and aerial part for the determination of the mass of the dry matter, height, leaf area, diameter of the stem and content of nutritious. The fertilization with $\mathrm{N}$, $\mathrm{P}$ and $\mathrm{K}$ provided larger growth and larger accumulation of $\mathrm{N}, \mathrm{P}$ and $\mathrm{K}$ in the aerial part and in the roots of the orange tree seedlings, in substratum of Pinus bark and vermiculite. In the orange tree 'Valencia' grafted on lemon tree citrumelo 'Swingle' the half of the recommended dose of $\mathrm{N}\left(459 \mathrm{mg} \mathrm{dm}^{-3}\right)$ and, the recommended dose of $\mathrm{P}$ and $\mathrm{K}\left(184\right.$ and $\left.876 \mathrm{mg} \mathrm{dm}^{-3}\right)$, respectively, provided better growth of the aerial part of the seedlings; however, in the recommended dose of $\mathrm{N}$ of $918 \mathrm{mg} \mathrm{dm}^{-3}$, it happened larger growth of the root system.

Index terms: N P K, mineral nutrition, orange seedlings, Citrus ssp.

'(Trabalho 237-07).Recebido em: 03-10-2007. Aceito para publicação em: 07-07-2008

${ }^{2}$ Professor Doutor, Depto. de Solos e Adubos, Universidade Estadual Paulista, Faculdade de Ciências Agrárias e Veterinárias (FCAV/Unesp). Via de Acesso Prof. Paulo Donato Castellane, s/n. 14870-000, Jaboticabal-SP. Bolsista CNPq. E-mail: rmprado@fcav.unesp.br

${ }^{3}$ Engenheiro Agrônomo, Doutorando, Depto. Solos e Adubos, FCAV/Unesp. Bolsista FAPESP. E-mail: danilorozane@yahoo.com.br

${ }^{4}$ Graduando em Agronomia da FCAV/Unesp. E-mail: gustavo.toru@ @otmail.com

${ }^{5}$ Engenheiro Agrônomo, Mestrando, Dept ${ }^{\circ}$ Solos e Adubos, FCAV/Unesp. E-mail: marcusribeiro@uft.edu.br

${ }^{6}$ Professor Adjunto, Depto. de Solos e Adubos, FCAV/Unesp. Bolsista CNPq. E-mail: natale@fcav.unesp.br

${ }^{7}$ Professor Titular, Depto. Ciências Exatas, FCAV/Unesp. Bolsista CNPq. E-mail: jcbarbosa@fcav.unesp.br

${ }^{8}$ Doutor, Dept ${ }^{\circ}$. Solos e Adubos, FCAV/Unesp. E-mail: amaurib@yahoo.com.br

Rev. Bras. Frutic., Jaboticabal - SP, v. 30, n. 3, p. 812-817, Setembro 2008 


\section{INTRODUÇÃO}

O Brasil é o maior produtor mundial de laranjas e, também, o maior exportador de suco cítrico concentrado, com destaque para o Estado de São Paulo (Agrianual, 2006). Para a formação de pomares cítricos, são utilizadas, em geral, mudas enxertadas que apresentam as seguintes vantagens: precocidade, uniformidade de produção e de qualidade dos frutos, facilidade na colheita e nos tratos culturais, adaptação a diferentes tipos de solos, tolerância a determinadas moléstias e à seca (Pompeu Júnior, 1991). O porta-enxerto mais utilizado na citricultura brasileira é o de limoeiro 'Cravo' (Citrus limonia L. Osbeck), e o segundo porta-enxerto mais utilizado no Estado de São Paulo é o citrumeleiro 'Swingle'. Este é um híbrido originário dos Estados Unidos, resultante do cruzamento entre Citrus paradisi $\mathrm{x}$ Poncirus trifoliata, sendo amplamente utilizado devido a sua tolerância às doenças, à seca e boa longevidade, substituindo muitas vezes o porta-enxerto limoeiro 'Cravo'. Outro aspecto é a atual tendência de manutenção de a cotação de preços da laranja ser baseada no teor de sólidos solúveis do fruto, que é superior nos frutos de plantas enxertadas sobre o citrumelo 'Swingle'.

Quando atingem o ponto de enxertia, os porta-enxertos são transferidos do tubete para sacolas plásticas, onde são enxertados e são formadas as mudas. O tempo para a formação de uma muda cítrica, via enxertia, oscila entre 18 e 36 meses, dependendo do clima da região e do nível tecnológico do viveiro (Abou-Rawash et al., 1998). Porém, com o uso da fertirrigação e com a adequada nutrição das mudas, esse tempo pode diminuir, resultando em menor custo para a produção de mudas.

Além da dose adequada de fertilizante, o parcelamento da adubação é importante, uma vez que pode ocorrer lixiviação de nutrientes, principalmente de $\mathrm{N}$ e $\mathrm{K}$, devido às constantes irrigações e às pequenas dimensões dos recipientes (sacolas). Neste contexto, uma forma promissora de fornecimento de nutrientes para a produção de mudas é via fertirrigação, uma vez que outras formas de aplicação, como a adubação foliar, não têm exercido efeito significativo sobre o crescimento das plantas (Boaretto et al., 1999). Em regiões produtoras de diversas culturas no Estado de São Paulo, a fertirrigação vem ganhando adeptos, principalmente pela maior facilidade na aplicação dos nutrientes na época adequada, além de atender à exigência nutricional de cada variedade, em todas as fases do desenvolvimento da planta. $\mathrm{Na}$ literatura, são escassos estudos que avaliaram a resposta de mudas de citros, cultivadas em substrato inerte, em recipientes e em ambiente protegido à adubação com $\mathrm{N}, \mathrm{P}$ e $\mathrm{K}$, via fertirrigação, durante toda a fase de produção das mudas.

Neste estudo, objetivou-se avaliar o crescimento e o estado nutricional de mudas de laranjeira 'Valência', enxertada sobre citrumeleiro 'Swingle', em função de adubações com N, P e K.

\section{MATERIAL E MÉTODOS}

O experimento foi conduzido em viveiro telado na FCAV/ Unesp Câmpus Jaboticabal-SP, no período de novembro de 2005 a janeiro de 2007. Segundo a classificação de Köppen, o clima é do tipo Cwa, subtropical com inverno curto e seco, e verão quente e chuvoso, caracterizando duas estações distintas, na altitude de $601 \mathrm{~m}$

O viveiro estava coberto por filme plástico transparente e revestido, nas laterais, com tela de abertura de malha de $1 \mathrm{~mm}$, para impedir a entrada de afídeos. As bancadas com as mudas foram dispostas $30 \mathrm{~cm}$ acima do solo, seguindo recomendação de Carvalho \& Laranjeira (1994). Foram realizadas irrigações diárias durante a condução do experimento. O substrato utilizado para o cultivo, em tubete e nas sacolas, foi composto por casca de Pinus e vermiculita com granulometria fina, cuja caracterização química, realizada segundo método holandês $(1: 1,5)$ adaptado de Sonneveld \& Elderen (1994), revelou que: $\mathrm{CE}=1,5 \mathrm{dS} \mathrm{m}^{-1} ; \mathrm{pH}=$ 5,$9 ; \mathrm{N}_{\text {nitrato }}=2,4 \mathrm{mg} \mathrm{L}^{-1} ; \mathrm{N}_{\text {amônia }}=31,4 \mathrm{mg} \mathrm{L}^{-1} ; \mathrm{P}=16,3 \mathrm{mg} \mathrm{L}^{-1} ; \mathrm{K}=67,9$ $\mathrm{mgL}^{-1} ; \mathrm{Ca}=108,9 \mathrm{mgL}^{-1} ; \mathrm{Mg}=58,8 \mathrm{mgL}^{-1} ; \mathrm{S}=188,7 \mathrm{mgL}^{-1} ; \mathrm{Cl}=27,0$ $\mathrm{mg} \mathrm{L}^{-1} ; \mathrm{Na}=9,6 \mathrm{mg} \mathrm{L}^{-1} ; \mathrm{B}=0,1 \mathrm{mg} \mathrm{L}^{-1} ; \mathrm{Cu}=0,1 \mathrm{mg} \mathrm{L}^{-1} ; \mathrm{Fe}=0,2 \mathrm{mg}$ $\mathrm{L}^{-1} ; \mathrm{Mn}=1,3 \mathrm{mg} \mathrm{L}^{-1}, \mathrm{e} \mathrm{Zn}=0,1 \mathrm{mg} \mathrm{L}^{-1}$.

O delineamento experimental foi o inteiramente casualizado, em esquema fatorial $3^{3}+1$, sendo 3 fatores (nitrogênio, fósforo e potássio - NPK), 3 doses e uma testemunha (sem adubação), com 3 repetições, com uma planta por unidade experimental, totalizando assim 84 unidades experimentais.

Na primeira fase do projeto, foi verificado que as doses mais adequadas para a produção de porta-enxertos de citrumeleiro 'Swingle', em tubetes, foram de $920 \mathrm{mg} \mathrm{de} \mathrm{N} \mathrm{dm}^{-3} \mathrm{e} 790 \mathrm{mg}$ de K $\mathrm{dm}^{-3}$, e de $100 \mathrm{mg}$ de $\mathrm{P} \mathrm{dm}^{-3}$ de substrato (Rozane et al., 2008).

As sementes do porta-enxerto do limoeiro 'Swingle' foram semeadas em 21-11-2005, em tubetes de 2,8 cm de diâmetro e 12,3 $\mathrm{cm}$ de altura, perfurados na base, utilizando uma semente em cada tubete, sendo utilizadas duas fileiras laterais como bordadura. A adubação foi realizada com superfosfato triplo (44\% de $\mathrm{P}_{2} \mathrm{O}_{5}$ ), adicionado e homogeneizado ao substrato antes da semeadura, nitrato de amônio ( $34 \%$ de $\mathrm{N}$ ) e cloreto de potássio (60\% de $\mathrm{K}_{2} \mathrm{O}$ ), aplicadas via fertirrigações semanais de $2 \mathrm{~mL}$ de solução por planta. Além destes adubos foi aplicado $\mathrm{CaSO}_{4}$ na dose de $0,9 \mathrm{~g} \mathrm{dm}^{-3}$ de substrato e, mensalmente, foram feitas pulverizações foliares com solução de micronutrientes contendo: $\mathrm{B}=0,2 ; \mathrm{Mn}=0,5 \mathrm{e} \mathrm{Zn}=0,6 \mathrm{~g} \mathrm{~L}^{-1}$ (Bernardi et al., 2000b).

Aos 150 dias após a semeadura, os porta-enxertos de citrumeleiro 'Swingle' foram transplantados para sacolas de polietileno de $20 \mathrm{~cm}$ de largura $\times 40 \mathrm{~cm}$ de altura, com capacidade de $5 \mathrm{~L}$, preenchidas com $2,5 \mathrm{~kg}$ de substrato, aplicando-se combinações das seguintes doses de nutrientes em $\mathrm{mg}$ por $\mathrm{dm}^{3}$ de substrato: $\mathrm{N}_{1 / 2}: 459, \mathrm{~N}_{1}: 918$ e $\mathrm{N}_{2}: 1836 ; \mathrm{P}_{1 / 2}: 92, \mathrm{P}_{1}: 184$ e $\mathrm{P}_{2:}$ 368; $\mathrm{K}_{1 / 2}: 438, \mathrm{~K}_{1}: 876$ e $\mathrm{K}_{2}: 1752$. Sendo que as doses recomendadas para a produção de mudas de citros, nesta fase, são: 918, 184 e $876 \mathrm{mg} \mathrm{dm}^{-3}$ de substrato de $\mathrm{N}, \mathrm{P}$ e K, respectivamente (Boaventura, 2003).

Aos 100 dias após o transplantio para as sacolas, foi realizada a enxertia, com laranjeira 'Valência' (Citrus sinnensis L. Osbeck), pelo método da borbulhia, em " $\mathrm{T}$ " invertido, sendo feito o amarrio com fitilho plástico. Após 30 dias, realizou-se o forçamento da brotação da borbulha, através da decapitação dos porta-enxertos acima da enxertia e retirou-se o fitilho.

Após os porta-enxertos terem sido transferidos dos 
tubetes para as sacolas e durante a fase de desenvolvimento dos enxertos, utilizou-se solução nutritiva, via água de irrigação, para os tratamentos, com N, P e K e aplicação de quantidades equilibradas e uniformes em todos os tratamentos com os demais nutrientes, na seguinte composição: $\mathrm{Ca}=142 ; \mathrm{Mg}=45 ; \mathrm{S}=55 ; \mathrm{B}$ $=0,55 ; \mathrm{Cu}=0,13 ; \mathrm{Fe}=1,8 ; \mathrm{Mn}=0,54 ; \mathrm{Zn}=0,23 \mathrm{e} \mathrm{Mo}=0,10 \mathrm{~g}$ por 1.000 litros de solução, conforme indicação de Boaventura (2003). A fertirrigação foi realizada mediante três aplicações semanais, com volume de $200 \mathrm{~mL}$ de solução nutritiva por planta, totalizando $600 \mathrm{~mL}$ por planta, por semana (Boaventura, 2003). As fontes de nitrogênio, fósforo e potássio utilizadas foram o nitrato de cálcio, o fosfato monopotássico/MAP e o cloreto de potássio, respectivamente. Salienta-se que os cuidados com o manejo da solução e escolha das fontes de nutrientes seguiram as indicações de Furlani et al. (1999).

Aos 180 dias após a enxertia, 424 dias (aproximadamente 14 meses) após a semeadura dos porta-enxertos, foram realizadas as avaliações que constaram das seguintes determinações: a altura das plantas, a área foliar (aparelho LI-3100 área meter ${ }^{\circledR}$ ), o diâmetro do caule e a altura das plantas a partir do colo, massa seca (estufa a $67 \pm 3^{\circ} \mathrm{C}$ ) do sistema radicular e da parte aérea, e teores de nutrientes, utilizando a metodologia descrita por Bataglia et al. (1983).

Os dados obtidos foram submetidos a análises de variância e, quando o $\mathrm{F}$ foi significativo a $5 \%$, as médias foram comparadas pelo teste de Tukey, a $5 \%$ de probabilidade (SAS INSTITUTE, 1996).

\section{RESULTADOS E DISCUSSÃO}

A aplicação de N P K incrementou significativamente a altura, o diâmetro do caule, a área foliar, a massa seca das folhas, do caule e das raízes das mudas de laranjeira 'Valência', enxertada sobre citrumeleiro 'Swingle', comparado à testemunha sem adubação (Tabelas 1e 2). Observou-se interação significativa para todas as variáveis de crescimento, exceto para a massa seca do caule referente à interação $\mathrm{N} x \mathrm{~K}$. Assim, a interação NPK indica que os efeitos da aplicação de um nutriente no crescimento das plantas depende de outros nutrientes. A resposta das mudas de laranjeira às doses de $\mathrm{N}$ foram semelhantes entre os componentes do crescimento. A altura das plantas, diâmetro do caule, área foliar e massa seca das folhas apresentaram melhor desempenho quando foram utilizados $459 \mathrm{mg} \mathrm{dm}^{-3}$ de $\mathrm{N}$, metade da dose recomendada por Boaventura (2003), e a massa seca das raízes e do caule apresentaram maiores valores na dose indicada de $918 \mathrm{mg} \mathrm{dm}^{-3}$.

De forma geral, observando as interações (Tabela 2), o maior crescimento das mudas ocorreu empregando-se N (459 mg $\mathrm{dm}^{-3}$ ). Na dose de $1.836 \mathrm{mg} \mathrm{dm}^{-3}$ de $\mathrm{N}$, houve expressivo efeito supressor em todos os componentes da parte aérea, indicando menor exigência em $\mathrm{N}$ da laranjeira 'Valência' enxertada sobre o porta-enxerto citrumelo 'Swingle', do que as observadas por Bernardi et al. (2000a) com laranjeira 'Valência' enxertada sobre limoeiro 'Cravo'.

Esses resultados indicam que as mudas de citros apresentam respostas diferenciadas a $\mathrm{N}$ em relação às variedades, confirmando estudos de Maust \& Williamson (1994) e Decarlos Neto et al. (2002), que verificaram exigências nutricionais em $\mathrm{N}$ diferenciadas entre variedades. $\mathrm{O} \mathrm{N}$ é um dos elementos mais importantes na nutrição das mudas. Essas doses foram, ainda, superiores comparadas às doses recomendadas de $250 \mathrm{mg} \mathrm{dm}^{-3}$, via fertirrigação, por Maust \& Williamson (1994), na África do Sul, quando da utilização de substrato de casca de Pinus e vermiculita. Nesse aspecto, é importante utilizar doses adequadas de $\mathrm{N}$, visto que estudos indicam que doses elevadas de $\mathrm{N}$ podem ser prejudiciais, proporcionando redução do transporte de açúcares e suscetibilidade ao ataque de pragas (Smith, 1965; Bernardi et al., 2000a).

Considerando as interações $\mathrm{P}$ e $\mathrm{K}$, em relação à altura das plantas e à massa seca das folhas, em geral, verifica-se ligeira superioridade das doses de P e K de 184 e $876 \mathrm{mg} \mathrm{dm}^{-3}$, respectivamente, comparado às doses de 92 e $438 \mathrm{mg} \mathrm{dm}^{-3} \mathrm{de} \mathrm{Pe}$ $\mathrm{K}$, respectivamente, satisfazendo a recomendação de Boaventura (2003).

Neste contexto, mais estudos são necessários utilizando maior número de doses de $\mathrm{N}, \mathrm{P}$ e $\mathrm{K}$ para definir as doses mais adequadas para a produção de mudas com diversos portaenxertos, em substrato de casca de Pinus e vermiculita, dada a resposta positiva das mudas à adubação, em termos de crescimento mais rápido, possibilitando a formação de mudas em menor tempo.

A adubação com N, P e K proporcionou acúmulo de maiores quantidades desses nutrientes na parte aérea e nas raízes das mudas de laranjeira, comparado à testemunha sem adubação, havendo resposta diferencial das doses e suas interações (Tabelas 3 e 4). A exemplo dos componentes de desenvolvimento, maiores quantidades acumuladas de $\mathrm{N}$ na parte aérea foram verificadas na menor dose de $\mathrm{N}\left(459 \mathrm{mg} \mathrm{dm}^{-3}\right)$, e nas raízes ocorreu incremento na quantidade acumulada em função do aumento da adubação com $\mathrm{N}$, resultando em maior produção de massa seca do sistema radicular. Porém, há que se destacar o efeito supressor no acúmulo de $\mathrm{N}, \mathrm{P}$ e $\mathrm{K}$ na parte aérea, quando foram aplicados $1.836 \mathrm{mg} \mathrm{dm}^{-3} \mathrm{de} \mathrm{N}$, o dobro da dose recomendada por Boaventura (2003).

Bernardi et al. (2000b) verificaram efeito quadrático na relação entre dose aplicada e acúmulo de nitrogênio nas folhas, quando foram utilizadas doses altas deste nutriente em mudas de laranjeira 'Valência', enxertada sobre limoeiro 'Cravo'. Isso confirma os resultados obtidos, além de indicar que doses excessivas de nitrogênio têm efeito supressor no seu conteúdo na parte aérea, mostrando que a definição da dose adequada de $\mathrm{N}$ e o manejo da adubação nitrogenada são pré-requisitos para a formação e a obtenção de mudas adequadamente equilibradas nutricionalmente.

É importante destacar, ainda, que o maior acúmulo de $\mathrm{Ne}$ o crescimento do sistema radicular na dose de $918 \mathrm{mg} \mathrm{dm}^{-3}$, recomendada por Boaventura (2003), não refletiu em melhor crescimento e acúmulo de nutrientes na parte aérea das mudas. Considerando as interações, em geral, houve efeito supressor sobre o acúmulo de N, P e K na parte aérea. Em relação às doses de $\mathrm{P}$ e $\mathrm{K}$ e suas interações, de maneira geral, a dose recomendada por Boaventura (2003), de 184 e $876 \mathrm{mg} \mathrm{L}^{-1}$, resultou em maiores 
acúmulos de P e K na parte aérea (Tabela 4).

As interações entre N, P e K, além das doses, determinam a quantidade ideal acumulada na folha, comparativamente a estudos com nutrientes isolados, conforme estudos de Bernardi et al. (2000b), e, segundo Koo (1985), as interações são o fator predominante e de maior influência. Neste contexto, várias interações são relatadas na literatura (Chapman, 1968; Embleton et al., 1978; Bernardi et al., 2000b).

Neste estudo, houve interação inversa entre o $\mathrm{N}$ e o K, com o efeito do $\mathrm{N}$ predominante sobre o K, ou seja, o aumento na dose de $\mathrm{N}$ resultou em decréscimo no acúmulo de $\mathrm{K}$; porém, o aumento na dose de $\mathrm{K}$ não resultou em decréscimo no acúmulo de N. Entretanto, o aumento da dose de K resultou em aumento do acúmulo de K na parte aérea (Tabela 4). Essa interação entre
N e K também foi verificada por Bernardi et al. (2000b) em laranjeira 'Valência' enxertada sobre limoeiro 'Cravo', com a utilização de substrato de casca de Pinus, vermiculita e perlita.

Outra interação mencionada na literatura é a relação inversa do N e P (Reese \& Koo, 1975), tanto do N sobre o P quanto do P sobre o N (Bernardi et al., 2000b), em laranjeira 'Valência' enxertada sobre limoeiro 'Cravo', caracterizando o efeito de concentração quando plantas com teores baixos de $\mathrm{N}$ apresentarem altos teores de $\mathrm{P}$; e efeito de diluição quando houver excesso de $\mathrm{N}$ com ocorrência de teores baixos de $\mathrm{P}$, sendo assim difícil encontrar folhas com excesso de $\mathrm{N}$ e $\mathrm{P}$ ao mesmo tempo. Neste estudo, houve apenas interação inversa do $\mathrm{N}$ sobre o P e do P sobre o N, na metade da dose recomenda

TABELA 1 - Resumo da análise de variância (valor de F e CV) dos componentes de crescimento da laranjeira 'Valência', enxertada sobre citrumeleiro 'Swingle'

\begin{tabular}{|c|c|c|c|c|c|c|}
\hline $\begin{array}{c}\text { Causas de } \\
\text { variação }\end{array}$ & Altura & $\begin{array}{c}\text { Diâmetro } \\
\text { do caule }\end{array}$ & Área foliar & $\begin{array}{c}\text { Massa seca } \\
\text { das folhas }\end{array}$ & $\begin{array}{c}\text { Massa seca } \\
\text { do caule }\end{array}$ & $\begin{array}{c}\text { Massa seca } \\
\text { das raízes }\end{array}$ \\
\hline $\mathrm{N}$ & $25,96^{* *}$ & $40,86^{* *}$ & $221,77^{* * *}$ & $72,72^{* * *}$ & 62,31 & 46,36 \\
\hline $\mathbf{P}$ & $2,75^{\mathrm{ns}}$ & $16,43^{* *}$ & $120,42^{* *}$ & $1,46^{\mathrm{ns}}$ & 12,14 ** & 29,36 \\
\hline K & $2,59^{\mathrm{ns}}$ & $4,56^{*}$ & $103,40^{* * *}$ & $1,51^{\mathrm{ns}}$ & 9,40 & 7,64 \\
\hline$N \times P$ & $44,74^{* *}$ & $32,18^{*}$ & $147,79^{* *}$ & $24,34^{* *}$ & $53,66^{* * *}$ & $21,18^{* *}$ \\
\hline $\mathrm{N} \times \mathrm{K}$ & $20,43^{* *}$ & 31,25 & $64,46^{* *}$ & 26,96 & $2,24^{\mathrm{ns}}$ & $63,50^{* *}$ \\
\hline$P \times K$ & $25,89^{* *}$ & $10,63^{* *}$ & $13,49^{* *}$ & $7,98^{* * *}$ & $4,68^{* * *}$ & 72,56 \\
\hline $\mathrm{N} \times \mathrm{P} \times \mathrm{K}$ & $31,20^{* *}$ & $15,78^{*}$ & $141,17^{* *}$ & $16,82^{* *}$ & $24,42^{* *}$ & $13,98^{* *}$ \\
\hline Trat. $\mathrm{x}$ Test. & $185,51^{* * *}$ & $79,89^{* *}$ & $458,16^{* * *}$ & $110,62^{* * *}$ & 144,50 & $66,12^{* *}$ \\
\hline CV (\%) & 9,8 & 7,6 & 7,7 & 15,9 & 12,6 & 13,1 \\
\hline
\end{tabular}

**; * e ns - Significativo a 1 e $5 \%$ de probabilidade e não-significativo, respectivamente

TABELA 2- Altura, diâmetro do caule, área foliar, massa seca das folhas, do caule e das raízes de laranjeira 'Valência', enxertada sobre citrumeleiro 'Swingle', em função da interação das doses de adubação com N, P e K. Jaboticabal, 2007.

\begin{tabular}{|c|c|c|c|c|c|c|c|c|c|c|}
\hline & $\mathrm{P}_{1 / 2}{ }^{*}$ & $\mathrm{P}_{1}$ & $\mathrm{P}_{2}$ & $\mathrm{~K}_{1 / 2}{ }^{*}$ & $\mathrm{~K}_{1}$ & $\mathrm{~K}_{2}$ & & $\mathrm{~K}_{1 / 2}$ & $\mathrm{~K}_{1}$ & $\mathrm{~K}_{2}$ \\
\hline & & & & ----- Al & ura das plan & as, $\mathrm{cm}$--- & & & --------. & ---------- \\
\hline $\mathrm{N}_{1 / 2}{ }^{*}$ & $74,67 \mathrm{Aa}$ & $68,56 \mathrm{Aa}$ & $52,22 \mathrm{Bb}$ & $72,00 \mathrm{Aa}$ & $65,00 \mathrm{Aab}$ & $58,44 \mathrm{Ab}$ & $P_{1 / 2}$ & $53,89 \mathrm{Bb}$ & $68,00 \mathrm{Aa}$ & $50,00 \mathrm{Bb}$ \\
\hline $\mathrm{N}_{1}$ & $58,11 \mathrm{Bab}$ & $54,00 \mathrm{Bb}$ & $62,00 \mathrm{Aa}$ & $57,55 \mathrm{Ba}$ & $53,67 \mathrm{Ba}$ & $62,89 \mathrm{Aa}$ & $\mathrm{P}_{1}$ & $56,00 \mathrm{Bb}$ & $65,89 \mathrm{Aa}$ & $60,78 \mathrm{Aa}$ \\
\hline $\mathrm{N}_{2}$ & $39,11 \mathrm{Cb}$ & $60,11 \mathrm{Ba}$ & $63,56 \mathrm{Aa}$ & $46,56 \mathrm{Cb}$ & $64,56 \mathrm{Aa}$ & $51,67 \mathrm{Bb}$ & $\mathrm{P}_{2}$ & $66,22 \mathrm{Aa}$ & $49,33 \mathrm{Bb}$ & $62,22 \mathrm{Aa}$ \\
\hline & & & & ------Di & metro do ca & de, cm--- & & & & \\
\hline $\mathrm{N}_{1 / 2}$ & $10,76 \mathrm{Aa}$ & $9,18 \mathrm{Ab}$ & $8,52 \mathrm{Cb}$ & $10,58 \mathrm{Aa}$ & $8,16 \mathrm{Bb}$ & $9,72 \mathrm{Aa}$ & $\mathrm{P}_{1 / 2}$ & 9,56Aa & $9,52 \mathrm{Aa}$ & $9,07 \mathrm{Aa}$ \\
\hline $\mathrm{N}_{1}$ & $10,10 \mathrm{Aa}$ & $8,93 \mathrm{Ab}$ & $8,85 \mathrm{Bb}$ & $9,04 \mathrm{Ba}$ & $9,12 \mathrm{Aa}$ & $9,72 \mathrm{Aa}$ & $\mathrm{P}_{1}$ & 8,89Aa & $8,78 \mathrm{ABa}$ & $7,40 \mathrm{Bb}$ \\
\hline $\mathrm{N}_{2}$ & $7,30 \mathrm{Bb}$ & $6,96 \mathrm{Bb}$ & $9,67 \mathrm{Aa}$ & $8,05 \mathrm{Ca}$ & $9,24 \mathrm{Aa}$ & $6,64 \mathrm{Bb}$ & $\mathrm{P}_{2}$ & $9,21 \mathrm{Aab}$ & $8,22 \mathrm{Bb}$ & $9,61 \mathrm{Aa}$ \\
\hline & & & & & rea foliar, & $\mathrm{m}^{2}$ & & & & \\
\hline $\mathrm{N}_{1 / 2}$ & $1240 \mathrm{Aab}$ & $1577 \mathrm{Aa}$ & $934 \mathrm{Bc}$ & 1319Aa & $1187 \mathrm{Ba}$ & $1245 \mathrm{Aa}$ & $\mathrm{P}_{1 / 2}$ & $661 \mathrm{Bb}$ & $942 \mathrm{Ca}$ & $1029 \mathrm{Ba}$ \\
\hline $\mathrm{N}_{1}$ & $916 \mathrm{Bb}$ & $1003 \mathrm{Bb}$ & $1518 \mathrm{Aa}$ & $789 \mathrm{Bb}$ & $1329 \mathrm{Aa}$ & 1319Aa & $\mathrm{P}_{1}$ & 996Ac & $1413 \mathrm{Aa}$ & $1169 \mathrm{Ab}$ \\
\hline $\mathrm{N}_{2}$ & $476 \mathrm{Cb}$ & 999Ba & $962 \mathrm{Ba}$ & $582 \mathrm{Cc}$ & $1085 \mathrm{Ca}$ & $770 \mathrm{Bb}$ & $\mathrm{P}_{2}$ & $1032 \mathrm{Ab}$ & $1246 \mathrm{Ba}$ & $1136 \mathrm{Aab}$ \\
\hline $\mathrm{N}_{1 / 2}$ & $12,62 \mathrm{Aa}$ & $12,47 \mathrm{Aa}$ & $8,59 \mathrm{Ab}$ & $13,60 \mathrm{Aa}$ & $10,98 \mathrm{Ab}$ & $9,09 \mathrm{Ab}$ & $\mathrm{P}_{1 / 2}$ & 7,61Ba & $9,54 \mathrm{a}$ & $9,64 \mathrm{Aa}$ \\
\hline $\mathrm{N}_{1}$ & 7,91Bb & $5,74 \mathrm{Cc}$ & $9,56 \mathrm{Aa}$ & $5,19 \mathrm{Cb}$ & $9,72 \mathrm{Aa}$ & $8,30 \mathrm{Aa}$ & $\mathrm{P}_{1}$ & $9,05 \mathrm{ABa}$ & $8,74 \mathrm{Aa}$ & $8,74 \mathrm{Aa}$ \\
\hline $\mathrm{N}_{2}$ & $6,26 \mathrm{Cb}$ & $8,32 \mathrm{Ba}$ & $6,90 \mathrm{Bab}$ & $7,45 \mathrm{Ba}$ & $6,30 \mathrm{Ba}$ & $7,72 \mathrm{Aa}$ & $\mathrm{P}_{2}$ & $9,59 \mathrm{Aa}$ & $8,73 \mathrm{Aab}$ & $6,74 \mathrm{Bb}$ \\
\hline $\mathrm{N}_{1 / 2}$ & $10,09 \mathrm{Aa}$ & $9,16 \mathrm{Aa}$ & $5,04 \mathrm{Cb}$ & - & - & - & $\mathrm{P}_{1 / 2}$ & $8,21 \mathrm{Aa}$ & $8,54 \mathrm{Ba}$ & $8,43 \mathrm{Aa}$ \\
\hline $\mathrm{N}_{1}$ & $10,61 \mathrm{Aa}$ & $8,00 \mathrm{Bb}$ & 9,99Aa & - & - & - & $\mathrm{P}_{1}$ & $7,69 \mathrm{Ab}$ & $10,02 \mathrm{Aa}$ & $7,74 \mathrm{ABb}$ \\
\hline $\mathrm{N}_{2}$ & $4,48 \mathrm{Bc}$ & $8,29 \mathrm{ABa}$ & $6,87 \mathrm{Bb}$ & - & - & - & $\mathrm{P}_{2}$ & 7,51 Aa & 7,61Ba & $6,78 \mathrm{Ba}$ \\
\hline$N_{1 / 2}$ & $3,02 \mathrm{Cb}$ & $5,39 \mathrm{Ba}$ & $5,60 \mathrm{Ba}$ & 7,47Aa & $2,52 \mathrm{Cc}$ & $\begin{array}{l}\text { lizes, g-- } \\
4,02 \mathrm{Cb}\end{array}$ & $P_{1 / 2}$ & $3,13 \mathrm{Cb}$ & 5,79Aa & $6,05 \mathrm{Aa}$ \\
\hline $\mathrm{N}_{1}$ & $6,43 \mathrm{Aa}$ & $6,16 \mathrm{Ba}$ & $6,66 \mathrm{Aa}$ & $5,18 \mathrm{Cb}$ & 7,30Aa & $6,76 \mathrm{Aa}$ & $\mathrm{P}_{1}$ & $9,52 \mathrm{Aa}$ & $5,30 \mathrm{Aab}$ & $4,79 \mathrm{Bb}$ \\
\hline $\mathrm{N}_{2}$ & $5,51 \mathrm{Bb}$ & $8,06 \mathrm{Aa}$ & $5,35 \mathrm{Bb}$ & $6,07 \mathrm{Bab}$ & $7,21 \mathrm{Ba}$ & $5,64 \mathrm{Bb}$ & $\mathrm{P}_{2}$ & $6,07 \mathrm{Ba}$ & $5,95 \mathrm{Aa}$ & $5,59 \mathrm{ABa}$ \\
\hline
\end{tabular}

Médias seguidas da mesma letra,maiúscula na coluna e minúscula na linha, não diferem estatisticamente entre si, pelo teste de Tukey, a $5 \%$ de probabilidade. - Não houve interação.* $\mathrm{N}_{1 / 2}: 459, \mathrm{~N}_{1}: 918$ e $\mathrm{N}_{2}: 1836 \mathrm{mg} \mathrm{dm}^{-3} . \quad \mathrm{P}_{1 / 2}: 92, \mathrm{P}_{1}: 184 \mathrm{e} \mathrm{P}_{2:} 368 \mathrm{mg} \mathrm{dm}^{-3 .} \quad \mathrm{K}_{1 / 2}: 438, \mathrm{~K}_{1}: 876$ e $\mathrm{K}_{2}: 1752 \mathrm{mg} \mathrm{dm}^{-3}$ 
TABELA 3- Resumo da análise de variância (valor de F e CV) do acúmulo de nutrientes na massa seca de raízes e da parte aérea da laranjeira 'Valência', enxertada sobre citrumeleiro 'Swingle'.

\begin{tabular}{|c|c|c|c|c|c|c|}
\hline \multirow{2}{*}{$\begin{array}{l}\text { Causas de } \\
\text { variação }\end{array}$} & \multicolumn{3}{|c|}{ Parte aérea } & \multicolumn{3}{|c|}{ Raízes } \\
\hline & $\mathrm{N}$ & $\mathrm{P}$ & $\mathrm{K}$ & $\mathrm{N}$ & $\mathrm{P}$ & $\mathrm{K}$ \\
\hline $\mathrm{N}$ & $3,96^{*}$ & $27,58^{* *}$ & $131,41^{* *}$ & $166,18^{* *}$ & $29,03^{* *}$ & $16,91^{* *}$ \\
\hline $\mathrm{P}$ & $4,69^{*}$ & $6,22^{* *}$ & $41,68^{* *}$ & $39,01^{* *}$ & $31,73^{* *}$ & 21,30 ** \\
\hline K & $5,02^{* *}$ & $6,85^{* *}$ & $56,88^{* *}$ & $0,01^{\mathrm{ns}}$ & $9,40^{* *}$ & $4,17^{*}$ \\
\hline $\mathrm{N} \times \mathrm{P}$ & $65,67^{* *}$ & $55,95^{* *}$ & $47,53^{* *}$ & $26,02^{* *}$ & $18,56^{* *}$ & $28,11^{* *}$ \\
\hline $\mathrm{N} \times \mathrm{K}$ & $12,71^{* *}$ & $7,93^{* *}$ & $7,35^{* *}$ & $38,68^{* *}$ & $34,02^{* *}$ & $44,33^{* *}$ \\
\hline $\mathrm{P} \times \mathrm{K}$ & $10,75^{* *}$ & $9,30^{* *}$ & $18,55^{* *}$ & $38,76^{* *}$ & $56,71^{* *}$ & $50,54^{* *}$ \\
\hline $\mathrm{N} \times \mathrm{P} \times \mathrm{K}$ & $30,84^{* *}$ & $33,22^{* *}$ & $21,57^{* *}$ & $7,11^{* *}$ & $11,04^{* *}$ & $9,06^{* *}$ \\
\hline Trat. x Test. & $212,53^{* *}$ & $228,87^{* *}$ & $182,51^{* *}$ & $101,39^{* *}$ & $47,08^{* *}$ & $52,43^{* *}$ \\
\hline CV (\%) & 11,5 & 10,8 & 12,4 & 15,0 & 15,2 & 16,8 \\
\hline
\end{tabular}

**; * e ns - Significativo a 1 e $5 \%$ de probabilidade e não-significativo, respectivamente

TABELA 4-Acúmulo de $\mathrm{N}, \mathrm{P}$ e K (mg por planta) na parte aérea e nas raízes das mudas de laranjeira 'Valência', enxertadas sobre citrumeleiro 'Swingle', em função de adubações com doses de N, P e K. Jaboticabal, 2007.

\begin{tabular}{|c|c|c|c|c|c|c|c|c|c|c|}
\hline & $\mathrm{P}_{1 / 2}{ }^{*}$ & $\mathrm{P}_{1}$ & $\mathrm{P}_{2}$ & $\mathrm{~K}_{1 / 2}{ }^{*}$ & $\mathrm{~K}_{1}$ & $\mathrm{~K}_{2}$ & & $\mathrm{~K}_{1 / 2}$ & $\mathrm{~K}_{1}$ & $\mathrm{~K}_{2}$ \\
\hline \multicolumn{11}{|c|}{ Acúmulo de nutrientes na parte aérea } \\
\hline & $\mathrm{P}_{1 / 2}$ & $\mathrm{P}_{1}$ & $\mathrm{P}_{2}$ & $\mathrm{~K}_{1 / 2}$ & $\mathrm{~K}_{1}$ & $\mathrm{~K}_{2}$ & & $\mathrm{~K}_{1 / 2}$ & $\begin{array}{c}\mathrm{K}_{1} \\
\end{array}$ & $\mathrm{~K}_{2}$ \\
\hline $\mathrm{N}_{1 / 2}{ }^{*}$ & $479,6 \mathrm{Aa}$ & $415,1 \mathrm{Ab}$ & & $420,1 \mathrm{Aa}$ & 393,7Aab & $324,5 \mathrm{Bb}$ & $\mathrm{P}_{1 / 2}$ & $373,4 \mathrm{~A}$ & $357,5 \mathrm{~B}$ & \\
\hline $\mathrm{N}_{1}$ & $375,7 \mathrm{Ba}$ & $281,6 \mathrm{Bb}$ & $420,8 \mathrm{Aa}$ & $298,7 \mathrm{Cb}$ & $393,3 \mathrm{Aa}$ & $386,2 \mathrm{Aa}$ & $\mathrm{P}_{1}$ & $330,6 \mathrm{Ab}$ & $422,9 \mathrm{Aa}$ & $348,8 \mathrm{Bab}$ \\
\hline $\mathrm{N}_{2}$ & $274,2 \mathrm{Cb}$ & $405,6 \mathrm{Aa}$ & $367,6 \mathrm{Ba}$ & $360,2 \mathrm{Ba}$ & $356,9 \mathrm{Aa}$ & $330,2 \mathrm{Ba}$ & $\mathrm{P}_{2}$ & $375,0 \mathrm{Aa}$ & $363,6 \mathrm{Bab}$ & $293,4 \mathrm{Cb}$ \\
\hline $\mathrm{N}_{1 / 2}$ & $46,6 \mathrm{Aa}$ & $45,1 \mathrm{Aa}$ & $28,7 \mathrm{Ab}$ & $43,4 \mathrm{Aa}$ & $41,9 \mathrm{Aab}$ & $35,1 \mathrm{ABb}$ & $\mathrm{P}_{1 / 2}$ & $35,2 \mathrm{Aa}$ & $37,0 \mathrm{Ba}$ & $39,8 \mathrm{Aa}$ \\
\hline $\mathrm{N}_{1}$ & $39,2 \mathrm{Bb}$ & $31,1 \mathrm{Cc}$ & $44,9 \mathrm{Aa}$ & $34,9 \mathrm{Bb}$ & $43,0 \mathrm{Aa}$ & $37,4 \mathrm{Aab}$ & $\mathrm{P}_{1}$ & $38,2 \mathrm{Aa}$ & $41,4 \mathrm{Aa}$ & $36,8 \mathrm{Aa}$ \\
\hline $\mathrm{N}_{2}$ & $26,1 \mathrm{Cc}$ & $40,2 \mathrm{Ba}$ & $31,7 \mathrm{Ab}$ & $33,3 \mathrm{Ba}$ & $32,0 \mathrm{Ba}$ & $32,8 \mathrm{Ba}$ & $\mathrm{P}_{2}$ & $38,2 \mathrm{Aa}$ & $38,6 \mathrm{ABa}$ & $28,7 \mathrm{Bb}$ \\
\hline $\mathrm{N}_{1 / 2}$ & $848,4 \mathrm{Aa}$ & $753,5 \mathrm{Ab}$ & $441,4 \mathrm{Bc}$ & $624,2 \mathrm{Ab}$ & $673,1 \mathrm{Aab}$ & $746,1 \mathrm{Aa}$ & $\mathrm{P}_{1 / 2}$ & $473,3 \mathrm{Ac}$ & $601,5 \mathrm{Ab}$ & $785,5 \mathrm{Aa}$ \\
\hline $\mathrm{N}_{1}$ & $664,8 \mathrm{Ba}$ & $443,8 \mathrm{Bb}$ & $596,6 \mathrm{Aa}$ & $396,5 \mathrm{Bc}$ & $586,9 \mathrm{Bb}$ & $721,9 \mathrm{Aa}$ & $\mathrm{P}_{1}$ & $415,8 \mathrm{Ac}$ & $576,2 \mathrm{Ab}$ & $698,8 \mathrm{Ba}$ \\
\hline $\mathrm{N}_{2}$ & $347,0 \mathrm{Cb}$ & $493,5 \mathrm{Ba}$ & $339,0 \mathrm{Cb}$ & $329,2 \mathrm{Bb}$ & $396,2 \mathrm{Cab}$ & $454,2 \mathrm{Ba}$ & $\mathrm{P}_{2}$ & $460,8 \mathrm{~A}$ & $478,4 \mathrm{~B}$ & $437,9 \mathrm{C}$ \\
\hline \multicolumn{11}{|c|}{ Acúmulo de nutrientes nas raízes } \\
\hline $\mathrm{N}_{1 / 2}$ & $55,5 \mathrm{Bb}$ & $95,9 \mathrm{Ca}$ & $104,4 \mathrm{Ca}$ & $134,7 \mathrm{Ba}$ & $43,8 \mathrm{Cb}$ & $77,2 \mathrm{Cb}$ & $P_{1 / 2}$ & $64,6 \mathrm{Cb}$ & $122,8 \mathrm{Aa}$ & $136,1 \mathrm{Aa}$ \\
\hline $\mathrm{N}_{1}$ & $131,3 \mathrm{Aa}$ & $123,2 \mathrm{Ba}$ & $138,8 \mathrm{Ba}$ & $106,8 \mathrm{Cb}$ & $146,6 \mathrm{Ba}$ & $139,9 \mathrm{Bab}$ & $P_{1}$ & $202,0 \mathrm{Aa}$ & $134,2 \mathrm{Ab}$ & $125,7 \mathrm{Ab}$ \\
\hline $\mathrm{N}_{2}$ & $136,7 \mathrm{Ac}$ & $242,7 \mathrm{Aa}$ & $163,9 \mathrm{Ab}$ & $156,9 \mathrm{Ab}$ & $207,2 \mathrm{Aa}$ & $179,1 \mathrm{Aab}$ & $\mathrm{P}_{2}$ & $131,8 \mathrm{Ba}$ & $140,7 \mathrm{Aa}$ & $134,5 \mathrm{Aa}$ \\
\hline $\mathrm{N}_{1 / 2}$ & $6,7 \mathrm{Bb}$ & $13,4 \mathrm{Ba}$ & $15,0 \mathrm{ABa}$ & $17,7 \mathrm{Aa}$ & $6,8 \mathrm{Bb}$ & $10,6 \mathrm{Bb}$ & $\mathrm{P}_{1 / 2}$ & $7,5 \mathrm{Cb}$ & $13,5 \mathrm{Aa}$ & $14,0 \mathrm{Aa}$ \\
\hline $\mathrm{N}_{1}$ & $15,3 \mathrm{Aa}$ & $15,1 \mathrm{Ba}$ & $16,4 \mathrm{Aa}$ & $13,5 \mathrm{Bb}$ & $17,7 \mathrm{Aa}$ & $15,7 \mathrm{Ab}$ & $\mathrm{P}_{1}$ & $24,0 \mathrm{Aa}$ & $13,2 \mathrm{Aab}$ & $11,1 \mathrm{Bb}$ \\
\hline $\mathrm{N}_{2}$ & $13,0 \mathrm{Ab}$ & $19,8 \mathrm{Aa}$ & $13,5 \mathrm{Bb}$ & $15,8 \mathrm{ABa}$ & $17,2 \mathrm{Aa}$ & $13,4 \mathrm{Aa}$ & $\mathrm{P}_{2}$ & $15,4 \mathrm{Ba}$ & $15,0 \mathrm{Aa}$ & $14,4 \mathrm{Aa}$ \\
\hline $\mathrm{N}_{1 / 2}$ & $96,5 \mathrm{Cb}$ & $186,4 \mathrm{Ba}$ & $189,9 \mathrm{Aa}$ & $234,0 \mathrm{Aa}$ & $82,9 \mathrm{Cc}$ & $156,0 \mathrm{Bb}$ & $\mathrm{P}_{1 / 2}$ & $93,0 \mathrm{Cb}$ & $170,3 \mathrm{Aa}$ & $206,6 \mathrm{Aa}$ \\
\hline $\mathrm{N}_{1}$ & $226,9 \mathrm{Aa}$ & $190,8 \mathrm{Bb}$ & $182,4 \mathrm{Ab}$ & $143,5 \mathrm{Bb}$ & $222,0 \mathrm{Aa}$ & $234,6 \mathrm{Aa}$ & $\mathrm{P}_{1}$ & $293,4 \mathrm{Aa}$ & $153,4 \mathrm{Ab}$ & $163,3 \mathrm{Bb}$ \\
\hline $\mathrm{N}_{2}$ & $146,7 \mathrm{Bb}$ & $232,9 \mathrm{Aa}$ & $116,9 \mathrm{Bb}$ & $167,8 \mathrm{Ba}$ & $179,6 \mathrm{Ba}$ & $149,1 \mathrm{Ba}$ & $\mathrm{P}_{2}$ & $158,9 \mathrm{Ba}$ & $160,7 \mathrm{Aa}$ & $169,6 \mathrm{Ba}$ \\
\hline
\end{tabular}

Médias seguidas da mesma letra, maiúscula na coluna e minúscula na linha, não diferem estatisticamente entre si, pelo teste de Tukey, a $5 \%$ de probabilidade

* $\mathrm{N}_{1 / 2}: 459, \mathrm{~N}_{1}: 918$ e $\mathrm{N}_{2}: 1836 \mathrm{mg} \mathrm{dm}^{-3}$

$\mathrm{P}_{1 / 2}: 92, \mathrm{P}_{1}: 184$ e $\mathrm{P}_{2}, 368 \mathrm{mg} \mathrm{dm}^{-3}$

$\mathrm{K}_{1 / 2}: 438, \mathrm{~K}_{1}: 876$ e $\mathrm{K}_{2}: 1752 \mathrm{mg} \mathrm{dm}^{-3}$

Rev. Bras. Frutic., Jaboticabal - SP, v. 30, n. 3, p. 812-817, Setembro 2008 


\section{CONCLUSÕES}

1- As adubações com $\mathrm{N}, \mathrm{P}$ e $\mathrm{K}$ proporcionaram maior crescimento e maior acúmulo de $\mathrm{N}, \mathrm{P}$ e $\mathrm{K}$ na parte aérea e nas raízes das mudas de laranjeira, em substrato de casca de Pinus e vermiculita, em relação à testemunha.

2- Na laranjeira 'Valência' enxertada sobre citrumeleiro 'Swingle', a dose de $459 \mathrm{mg} \mathrm{dm}^{-3}$ de N, e as doses de P e K $184 \mathrm{e}$ $876 \mathrm{mg} \mathrm{dm}^{-3}$, respectivamente, proporcionaram melhor crescimento da parte aérea das mudas; porém, na dose recomendada de $\mathrm{N}$, de $918 \mathrm{mg} \mathrm{dm}^{-3}$, ocorreu maior crescimento do sistema radicular.

\section{REFERÊNCIAS}

ABOU-RAWASH, M.; EL-WAKELL, H.F.; KASSEM, N.; MOHAMED, E.A. Studies on the vegetative propagation of some citrus rootstocks. Annals of Agricultural Science, Cairo, v.43, p.523-537, 1998 .

AGRIANUAL 2006: anuário da Agricultura Brasileira. São Paulo: FNP Consultoria e Comércio, 2005. p.257-270.

BATAGLIA, O.C.; FURLANI, A.M.C.; TEIXEIRA, J.P.F.; FURLANI, P.R.; GALLO, J.R. Métodos de análise química de plantas. Campinas: Instituto Agronômico, 1983. 48p. (Circular, 78)

BERNARDI, A.C.C.; CARMELLO, Q.A.C.; CARVALHO, S.A. Desenvolvimento de mudas de citros cultivadas em vaso em resposta à adubação NPK. Scientia Agricola, Piracicaba, v.57, p.733-738, 2000a.

BERNARDI, A.C.C.; CARMELLO, Q.A.C.; CARVALHO, S.A. Macronutrientes em mudas de citros cultivadas em vasos em resposta à adubação NPK. Scientia Agricola, Piracicaba, v.57, p.761-767, 2000b.

BOARETTO, A.E.; SCHIAVINATO NETO, P.; MURAOKA, T. Fertilização foliar de nitrogênio para laranjeira em estágio de formação. Scientia Agrícola, Piracicaba, v.56, p.621-626,1999.

BOAVENTURA, P.S.R. Demanda por nutrientes de portaenxertos e mudas cítricas produzidas em substrato em ambiente protegido. 2003. 63 f. Dissertação (Mestrado em Agricultura Tropical e Subtropical) - Universidade Estadual de Campinas, Campinas, 2003.

CARVALHO, S.A de; LARANJEIRA, F.F. Protótipo de viveiro de mudas certificadas e borbulheiras sob telado à prova de afídeos do Centro de Citricultura-IAC. Laranja, Cordeirópolis, v.15, p.213220, 1994.
CHAPMAN, H.D. The mineral nutrition of citrus. In: REUTER, V.; BATCHELOR, L.D.; WEBBER, H. (Ed.). The citrus industry. Berkeley: University of Califórnia, 1968. p.127-289.

DECARLOS NETO, A.; SIQUEIRA, D.L.; PEREIRA, P.R.G.; ALVAREZ V.H. Crescimento de porta-enxertos de citros em tubetes influenciados por doses de N. Revista Brasileira de Fruticultura, Jaboticabal, v.24, p.199-203, 2002.

EMBLETON, T.W.; JONES, W.W.; LABANAUSKAS, C.K.; REUTHER, W. Leaf analysis as a diagnostic tool and guide to fertilization. In: REUTHER, W. (Ed). The citrus industry. Berkely: University of California, 1973. v.3, cap.6, p.183-210.

FURLANI, P.R.; SILVEIRA, L.C.P.; BOLONHEZI, D.; FAQUIN, V. Cultivo hidropônico de plantas. Campinas: IAC, 1999. 52 p. (Boletim Técnico, 180)

KOO, R.C.J. Potassium nutrition of citrus. In: MUNSON, R.D. (Ed.) Potassium in agriculture. Madison: SSSA, 1985. p.10771086.

MAUST, B.E.; WILLIAMSON, J.G. Nitrogen nutrition of containerized citrus nursery plants. Journal of the American Society for Horticultural Science, Alexandria, v.119, p.195-201, 1994.

POMPEU JÚNIOR, J. Porta-enxertos. In: RODRIGUEZ, O. (Coord.). Citricultura brasileira. 2.ed. Campinas: Fundação Cargill, 1991, v.1, p.265-280.

REESE, R.L. ; KOO, R.C.J. Effects of N and K fertilization on leaf analysis, tree size and yield of three major Florida orange cultivars. Journal of the American Society for Horticultural Science, Alexandria, v.100, p.195-198, 1975.

ROZANE, D.E.; PRADO, R.de M.; NATALE, W.; BEUTLER, A.N.; SILVA, S.R.da; BARBOSA, J.C. Nitrogênio, fósforo e potássio na nutrição e produção de porta-enxerto de limoeiro citrumelo 'Swingle'. Revista Ceres, Viçosa, v.54, n.315, p.422-429, 2007.

SAS INSTITUTE. The SAS-system for windows: release 6.11 (software). Cary: Statistical Analysis System Institute, 1996.

SMITH, P.F. Effect of nitrogen source and placement on the root development of Valencia oranges trees. Proceedings of the Florida State Horticultural Society, Winter Haven, v.78, p.55-59, 1965.

SONNEVELD, C.; ELDEREN, C.W.van. Chemical analysis of peaty growing media by means of water extraction. Communications in Soil Science and Plant Analysis, Monticello, v.25, p. 31993208,1994 . 